\title{
Endoscopic ultrasound-guided transgastric drainage of radiologically inaccessible left lobe liver abscess involving segment 4, caudate lobe, and left lateral segments using a modified technique $\square$
}

두)(요 $\odot$

\author{
Authors \\ Sharad Chandra, Urvashi Chandra \\ Institution \\ Department of Gastroenterology, Meghraj Memorial \\ Hospital, Jhansi, Uttar Pradesh, India
}

submitted 27.7.2020

accepted after revision $\quad 30.9 .2020$

Bibliography

Endoscopy International Open 2021; 09: E35-E40

DOI 10.1055/a-1293-7746

ISSN 2364-3722

(C) 2021. The Author(s).

This is an open access article published by Thieme under the terms of the Creative Commons Attribution-NonDerivative-NonCommercial License, permitting copying and reproduction so long as the original work is given appropriate credit. Contents may not be used for commecial purposes, or adapted, remixed, transformed or built upon. (https://creativecommons.org/licenses/by-nc-nd/4.0/)

\author{
Corresponding author \\ Sharad Chandra, Meghraj Memorial - Gastroenterology, 95, \\ Civil Lines, Jhansi, Uttar Pradesh 284001, India \\ sharadurvashi@gmail.com
}

\section{ABSTRACT}

Liver abscess requiring drainage is conventionally managed by interventional radiology-guided percutaneous drainage (PCD). Radiologically inaccessible abscesses are managed with laparoscopic or open surgery, which carries high rates of morbidity and mortality.

EUS-guided transluminal liver abscess drainage is minimally invasive and can be an alternative approach for caudate lobe, segment 4, and left lateral segment abscesses. We report on three consecutive patients with radiologically inaccessible left lobe liver abscess involving the caudate lobe, segment 4, and lateral segment in whom EUS-guided transluminal drainage using a modified technique was successful.

\section{Introduction}

Radiologically-guided percutaneous drainage (PCD) is the standard of care for liver abscess requiring drainage. Inaccessible abscess is managed with laparoscopic or open surgery, which carries high rates of morbidity and mortality.

Endoscopic ultrasound (EUS) guidance allows easy access to radiologically inaccessible left lobe liver abscesses. EUS-guided transmural drainage can be a safe, effective, and minimally invasive alternative approach in such patients. We report on three consecutive patients with radiologically inaccessible complicated left lobe abscesses involving the caudate lobe, segment 4, and left lateral segments in whom EUS-guided transgastric transluminal drainage was successful.

\section{Patients and methods}

After receipt of written informed consent, EUS was performed using a therapeutic linear array echoendoscope (Olympus GFUCT180) and carbon dioxide insufflators under propofol sedation. A 19G needle (Echotip Ultra: Cook-Endoscopy) was introduced into the abscess cavity through the transgastric route, where there was a rim of intervening liver parenchyma of at least $1 \mathrm{~cm}$. This was done to avoid peritoneal spillage during drainage or accidental catheter dislodgement. Following aspiration, a 0.035-inch guidewire (Jagwire: Boston Scientific) was coiled in the abscess cavity, forming two rounds. Under fluoroscopic and EUS guidance, serial dilatation of the hepatico-gastrostomy was performed using a 6F Cystotome (Endoflex, Germany) followed by a 7F and 10F Sohendra dilator (Cook-Endoscopy). Active aspiration of the abscess cavity was done with a Sohendra dilator with flushing requirement standby if the catheter got blocked. A double pigtail stent and /or $8 \mathrm{~F}$ 


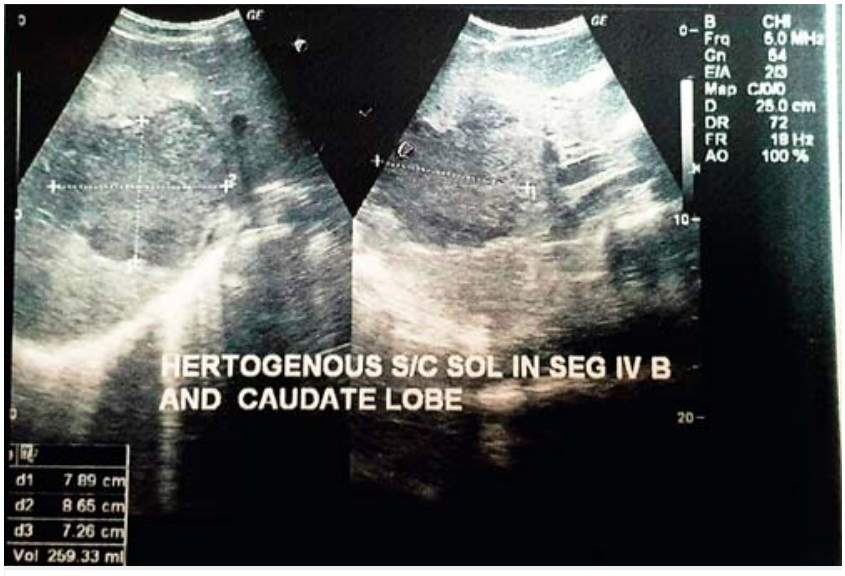

Fig. 1 Ultrasound showing heterogenous subcapsular abscess in segment $4 \mathrm{~b}$ and the caudate lobe, inaccessible to PCD.

or $10 \mathrm{~F}$ nasocystic drain (NCD) was then placed under fluoroscopic guidance for transluminal drainage. Thereafter, NCD was put on intermittent suction until there was spontaneous drainage aided by gravity

\section{Case 1}

A 30-year-old man presented with high-grade fever and abdominal pain for 2 weeks. Laboratory testing revealed a total leukocyte count of $33,400 / \mathrm{mm}^{3}$ with $90 \%$ neutrophils, serum creatinine $1.46 \mathrm{mg} / \mathrm{dL}$, and serum albumin $2.2 \mathrm{~g} / \mathrm{dL}$. Ultrasound showed a large, heterogenous subcapsular abscess in segment $4 \mathrm{~B}$ and the caudate lobe (volume $259 \mathrm{~mL}$ ) ( $\triangleright$ Fig. 1) with moderate ascites without any septae. Ascitic fluid aspiration showed frank pus. A diagnosis of ruptured liver abscess with peritonitis was considered.

The abscess was high risk for PCD as opined by an interventional radiologist because it was in the caudate lobe and segment 4B with intervening vessels in the path of access. The patient was given the option of surgery or EUS-guided transluminal drainage of the liver abscess, followed by PCD for intraperitoneal collection. The patient consented to EUS-guided drainage with surgery as a backup.

EUS-guided drainage was done with active aspiration of 160$\mathrm{mL}$, thick, anchovy-sauce pus suggestive of amoebic etiology. This was followed by placement of an $8 \mathrm{~F}$ transmural stent ( $\triangleright$ Video 1 and $\triangleright$ Fig. 2 and $\triangleright$ Fig. 3 ).

Interventional radiology-guided drains were then placed in the right and left paracolic gutter. Contents showed pus with a bilirubin of $16.64 \mathrm{mg} / \mathrm{dL}$. After drainage, the abscess cavity refilled from a volume of $90 \mathrm{~mL}$ to $138 \mathrm{~mL}$ within 48 hours. In addition, there was a high-volume bilious output from both paracolic gutter drains. The possibility of stent dysfunction and biliary communication of abscess was considered.

Repeat EUS-guided drainage with active aspiration of the contents and placement of an 8F NCD was done alongside the previously placed stent ( $\mathbf{F i g} \mathbf{4}$ ).

Following repeat drainage, the abscess cavity collapsed. However, NCD continued to drain a high volume of bile.

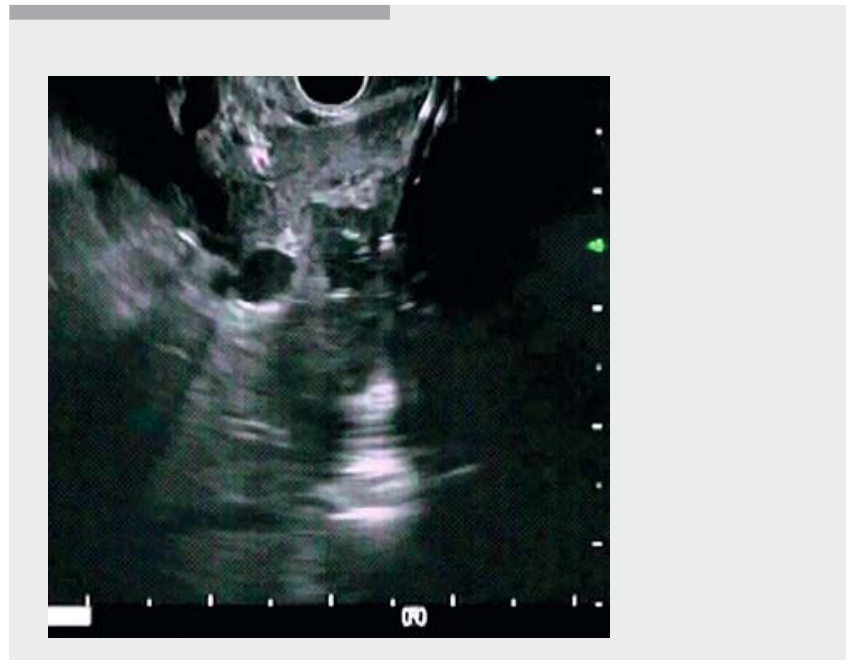

$\triangle$ Video 1 Large heterogenous abscess in the caudate lobe and segment 4 with peritoneal rupture. A 19G EUS needle was introduced into the abscess through the transgastric route, followed by coiling of 0.035 -inch guidewire in the abscess cavity. Serial dilatation was performed using a $6 \mathrm{~F}$ cystotome followed by a $7 \mathrm{~F}$ and $10 \mathrm{~F}$ Sohendra dilator. Active aspiration was done with a $10 \mathrm{~F}$ Sohendra dilator. An $8 \mathrm{~F}$ double pigtail stent was then deployed for transmural drainage.
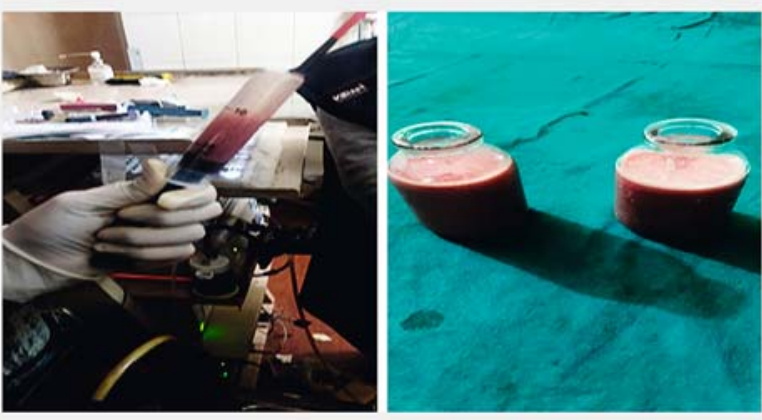

- Fig. 2 Active aspiration of anchovy sauce with 10F Sohendra dilator.

ERCP nasobiliary drain (NBD) placement was done bridging the biliary communication with the abscess cavity ( $\triangleright$ Fig. $\mathbf{5}$ ).

The patient received intravenous (IV) antibiotics for 2 weeks. NCD and paracolic gutter drains dried up on day 14. Because the NCD dye study showed no leakage, NCD was internalized into the stomach. Thereafter, the NBD was removed and the patient was discharged. The stent and NCD were removed after 3 months.

\section{Case 2}

A 24-year-old man presented with a high-grade fever and abdominal pain of 7 days duration. Abdominal ultrasound showed multiple liver abscesses. Segment 4B abscess (volume $70 \mathrm{~mL}$ ) had ruptured and was inaccessible to PCD as it was deep seated with intervening vasculature. The ruptured contents 


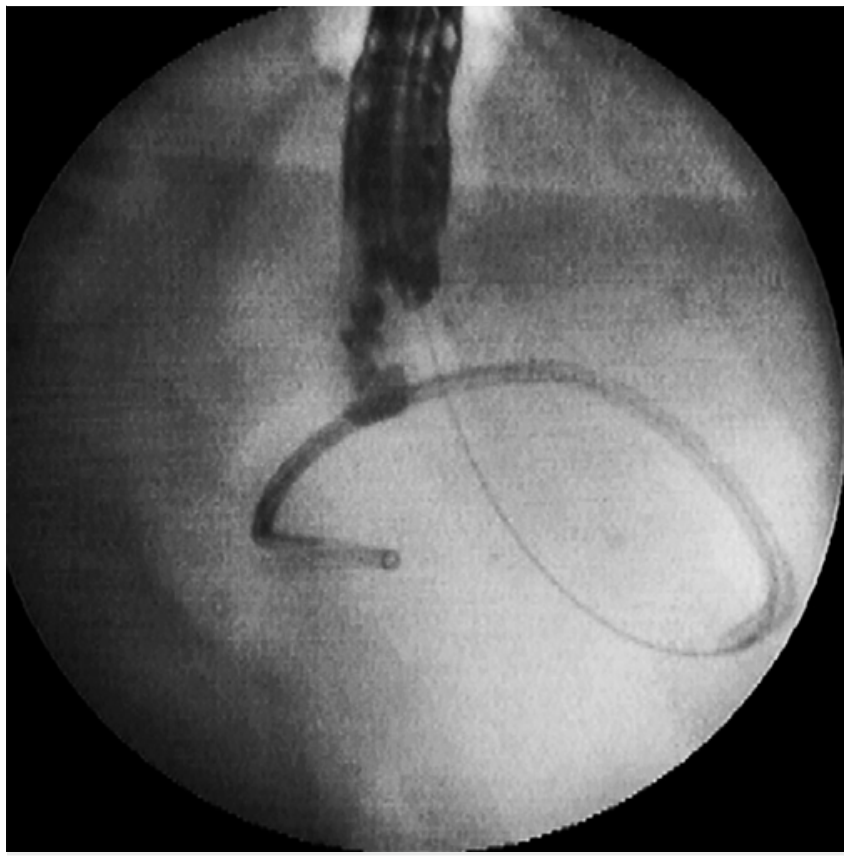

- Fig. 3 Fluoroscopic image showing deployment of double pigtail stent.

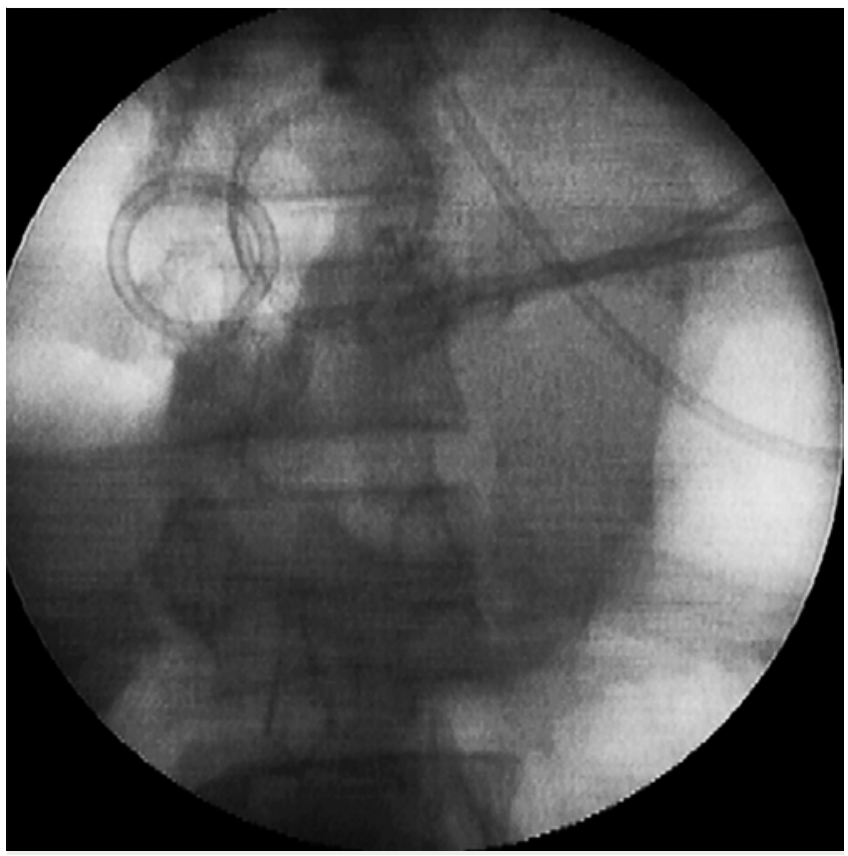

- Fig. 4 Fluoroscopic image showing 8F pigtail stent and 8F NCD placed through high lesser curve.

were localized in the perihepatic region. Laboratory examination revealed a hemoglobin $(\mathrm{Hgb})$ of $7.8 \mathrm{~g} / \mathrm{dL}$, total leukocyte count $41,800 / \mathrm{mm}^{3}$ with $97 \%$ neutrophils, serum creatinine $3.47 \mathrm{mg} / \mathrm{dL}$, serum bilirubin $4.92 \mathrm{mg} / \mathrm{dL}$ with direct serum bilirubin $4.38 \mathrm{mg} / \mathrm{dL}$ and serum alkaline phosphatase $425 \mathrm{IU}$.

A diagnosis of multiple liver abscesses with contained rupture of segment $4 \mathrm{~B}$ abscess, sepsis, and early organ dysfunction was considered. The patient was started on IV antibiotics. Be-

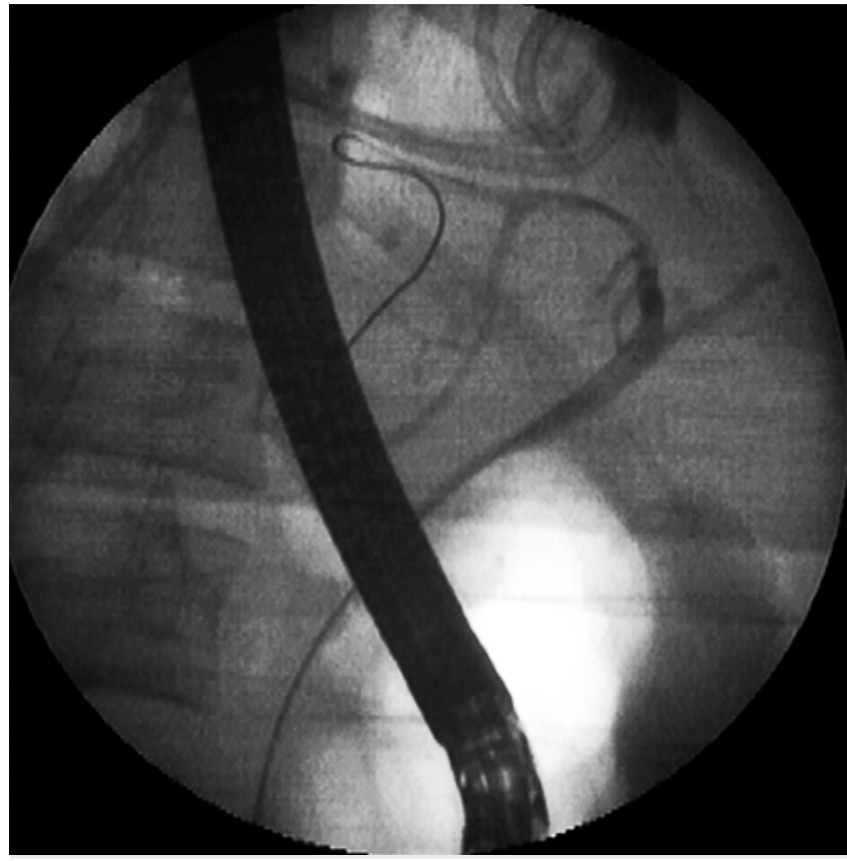

- Fig. 5 Fluoroscopic image of ERCP showing guidewire bridging the biliary communication with stent and NCD in situ.

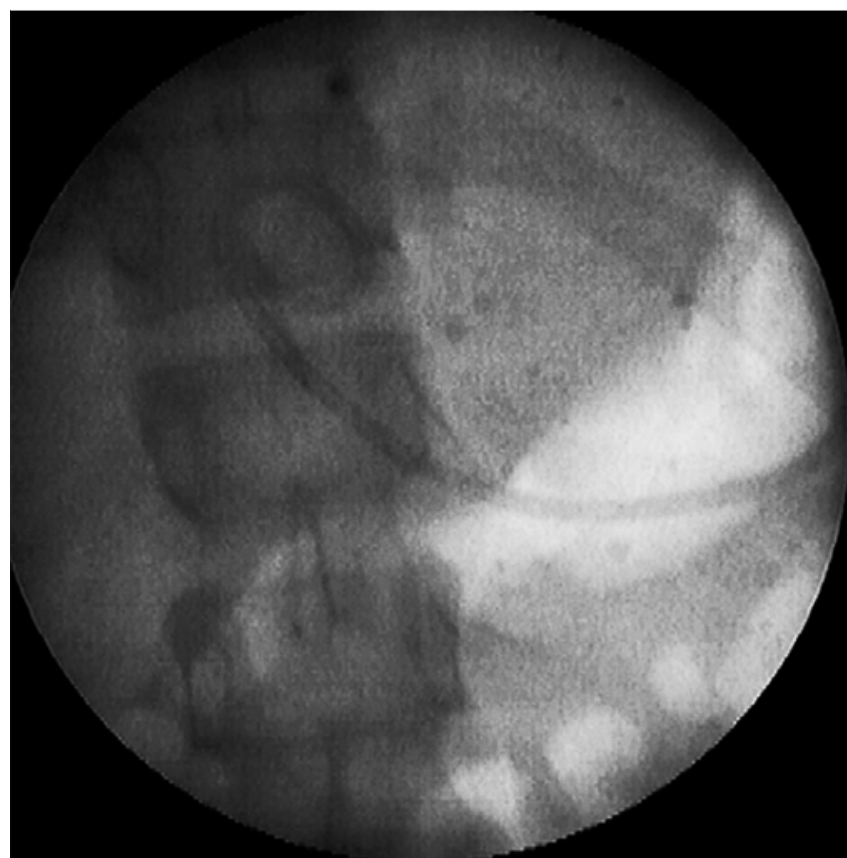

Pig. 6 Fluoroscopic image showing NCD placed transantrally.

cause the patient had sepsis, all abscesses required drainage. A hybrid approach of EUS-guided transgastric drainage with $10 \mathrm{~F}$ NCD placement in the ruptured segment 4 abscess ( Fig. 6) and percutaneous aspiration (PCA) of radiologically accessible abscesses was done. Transmural drainage was done as contents of the ruptured segment 4 abscess were thick and there was the possibility of biliary communication. 
Initially, the NCD output was anchovy sauce, which became bilious and then stopped over the next few days. The patient received IV antibiotics for 2 weeks. NCD was internalized at 7 days and removed after 8 weeks.

\section{Case 3}

A 55-year-old man presented with abdominal pain, vomiting, and fever for 10 days. Laboratory investigations revealed a $\mathrm{Hgb}$ of $9.6 \mathrm{~g} / \mathrm{dL}$, total leukocyte count $16,400 / \mathrm{mm}^{3}$ with $83 \%$ neutrophils, and a platelet counts of $90,000 / \mu \mathrm{L}$. Ultrasound showed multiple abscesses in the liver. Abscesses were seen in segments 4,5 , and 7 of $60 \mathrm{~mL}, 100 \mathrm{~mL}$, and $150 \mathrm{~mL}$, respectively. Another collection $(103 \mathrm{~mL})$ was seen in the perihepatic space from a contained rupture of the segment 4 abscess. In addition, there were communicating abscesses in segment 2 (vol $44 \mathrm{~mL}$ ) and segment 3 (vol $102 \mathrm{~mL}$ ) of the liver, which were inaccessible to PCD, owing to being deep seated with intervening vessels. A diagnosis of multiple liver abscesses with contained rupture was considered. EUS-guided aspiration or transmural drainage was planned for radiologically inaccessible and PCD or PCA for accessible abscesses. On EUS-guided aspiration, the contents were very thick. Therefore, transmural drainage with a 10F NCD placement was performed following active aspiration of $70 \mathrm{~mL}$ of thick anchovy sauce. PCD of the larger abscess and the other abscesses and perihepatic collection were done. The NCD aspirate turned bilious on Day 2 and stopped after 7 days. NCD was internalized on Day 7 . The patient received 2 weeks of antibiotic therapy.

\section{Discussion}

There are 12 case reports and three retrospective studies in the literature on EUS-guided drainage of liver abscess ( $>$ Table 1 ). The present case series of three complicated radiologically inaccessible left lobe abscesses involving the caudate lobe, segment 4, and left lateral segments further supports the role of EUS-guided transluminal drainage in such selected patients.

In all reported cases, a standard technique for EUS guided pseudocyst drainage was followed. However, there is no uniformity in the literature regarding the choice of endoprothesis for transluminal drainage. Double pigtail stents $7 \mathrm{~F}$ to $10 \mathrm{~F}$ and/or $5 \mathrm{~F}$ to 7F NCD were used as endoprostheses in the early reported cases $[1-6,9]$. In all recent publications, the authors have favored fully-covered self-expandable metal stent (FCSEMS) over plastic stents to achieve complete drainage and early abscess resolution $[7-8,10]$. The poblem with plastic stents is inadequate drainage. Although FCSEMS provides good drainage, it is not cost effective and has a risk of severe complications like stent migration, perforation and bleeding $[10,11]$.

The present case series has shown that a $10 \mathrm{~F}$ nasocystic drain in radiologically-inaccessible liver abscess works as effectively as PCD, and adding active aspiration of abscess improves drainage and clinical outcome.

NCD worked well as a prosthesis in all three patients. NCD makes drainage more predictable by allowing active aspiration and/or flushing if it gets blocked, and one can diagnose biliary communication by monitoring the drain output. The EUS scope allows deployment of a 10F drain and if required, the NCD can be upgraded to $12 \mathrm{~F}$. This upgrading technique facilitates guidewire access of the cavity with an endoscopic retrograde cholangiopancreatography (ERCP) scope, followed by placement of a 12 F NCD under fluoroscopic guidance. Guidewire access can be achieved alongside a 10F NCD. Another approach is through the NCD, by withdrawing the NCD retrogradely across the channel of the ERCP scope and using the NCD as a guide to slide the scope up to the transluminal drainage site. Thereafter, NCD is withdrawn maintaining access of the guidewire.

Placement of a 7F NCD alone was successful in draining large abscess $(11 \times 7 \mathrm{~cm})$ as reported by Seewald et al [1]. Koizumi et al [6] and Yamamoto et al [9] reported success with a 5F NCD. A 10F NCD was used in Cases 2 and 3, as it is equivalent to a standard PCD catheter and allows thick contents to drain.

None of our patients had any adverse events. As reported with PCD, there is a possibility of accidental self-dislodgement of the catheter, which can be repositioned if required. The NCD was removed after 6 weeks in all patients, allowing the tract to mature and the abscess cavity to collapse.

In the initial reported cases, the authors preferred using single or multiple double pigtail plastic stents of $7 \mathrm{~F}$ to $10 \mathrm{~F}$ for transmural drainage [2-5]. In Case 1, an 8F stent was deployed to drain the liver abscess. The stent got blocked due to thick residual contents and the cavity refilled due to biliary communication. Using a small-diameter plastic stent is associated with unpredictable drainage. Stents drain liquefied contents, however, if the contents are thick, the stents tend to get blocked. Therefore, plastic stents alone do not have much of a role in abscess drainage unless they are combined with NCD placement.

Placing NCD alongside the stent offers a wicking effect and provides the option of performing active lavage of abscess cavity. This worked in our Case 1. Successful transmural drainage with a stent and NCD placement has been reported by Itoi et al [4] in two cases and Noh et al in one [3]. A 10F NCD alone is sufficient, as seen in Cases 2 and 3, and there is no need to add stents and make the procedure more invasive.

In the present study to improve drainage, we improvised by adding the step of active aspiration of abscess contents with a $10 \mathrm{~F}$ Sohendra dilator. Active decompression of the abscess cavity lowers the chances of peritoneal spillage of contents and favors early cavity resolution. A dilator with a wide bore (distal end) up to 7F can allow aspiration of extremely thick contents if required. In large multiloculated abscesses, maneuvering the dilators to different sites under fluoroscopic guidance within abscess cavity can break loculation, allowing aspiration from multiple sites.

All recent publications have used FCSEMS or lumen-apposing metal stents (LAMS) for transmural drainage, as they allow rapid initial decompression of an abscess followed by predictable sustained drainage with minimal risk of peritoneal spillage. The same result was achieved with active aspiration with an Sohendra dilator followed by placement of a 10F NCD. The average median hospital stay reported following FCSEMS placement is 2 to 3 weeks $[7,8]$. In the case study presented, the hospital stay was 2 weeks for Case 1 and 1 week each for Cases 2 
- Table 1 Summary of literature on EUS-guided drainage of liver abscess [11].

\begin{tabular}{|c|c|c|c|c|}
\hline Author (year) & Abscess Number & Location & Drainage method & Approach \\
\hline Seewald et al. (2005) & 1 & Left lobe & $7 F N C D$ & TG \\
\hline Ang et al. (2009) & 1 & Left lobe & $8 \mathrm{~F}, 10 \mathrm{~F}$ pigtail PS & TG \\
\hline \multirow[t]{2}{*}{ Noh et al. (2010) } & 3 & Left lobe (1) & $7 F$ NCD & TG (2) \\
\hline & & Caudate lobe (2) & 7F pig tail PS & $\mathrm{TD}(1)$ \\
\hline \multirow[t]{2}{*}{ Itoi et al. (2011) } & 2 & Caudate lobe (1) & 7F straight and pigtail PS & TD \\
\hline & & Left lobe (1) & $5 F \mathrm{NCD}$ & TG \\
\hline \multirow[t]{2}{*}{ Keohane et al. (2011) } & 2 & Caudate lobe (2) & 7F pigtail PS & TG \\
\hline & & & 10F pigtail PS & TG \\
\hline Ivanina et al. (2012) & 1 & Caudate lobe & $(N / A) F N C D$ & TG \\
\hline Medrado et al. (2013) & 1 & Left lobe & FCSEMS & TG \\
\hline Alcaide et al. (2013) & 1 & Left lobe & LAMS & TG \\
\hline Kawakami et al. (2014) & 1 & Left lobe & FCSEMS & TG \\
\hline Koizumi et al. (2014) & 1 & Left lobe & $7 F$ NCD & TG \\
\hline Kodama et al. (2015) & 1 & Left lobe & FCSEMS & TG \\
\hline \multirow[t]{2}{*}{ Ogura et al. (2016) } & 8 & Left lobe (6) & FCSEMS & TG (6) \\
\hline & & Right lobe (2) & FCSEMS & $\mathrm{TD}(2)$ \\
\hline \multirow[t]{2}{*}{ Tonozuka et al. (2015) } & 7 & Left lobe (6) & FCSEMS & TG (6) \\
\hline & & Right lobe (1) & FCSEMS & $\mathrm{TD}(1)$ \\
\hline Yamamoto et al. (2017) & 1 & Right lobe & 5-F NCD & TD \\
\hline \multirow[t]{2}{*}{ Carbajo Lopez et al. (2019) } & 9 & Left lobe (3) & FCSEMS & TG (3) \\
\hline & & Right lobe (6) & LAMS (2) & TD (6) \\
\hline
\end{tabular}

and 3. The overall inference from the present case series is that NCD is cost effective compared with FCSEMS.

Biliary communication is reported in $6 \%$ to $10 \%$ patient with liver abscesses undergoing PCD and was present in all three cases. In Case 1, ERCP with sphincterotomy and NBD placement resulted in healing of biliary communication, while in the other two cases, drainage stopped with a conservative approach.

Case 1 is the first report in the literature of successful EUSguided drainage of a ruptured radiologically-inaccessible liver abscess with biliary communication causing peritonitis.

Cases 2 and 3 had multiple liver abscesses with contained rupture of one of the abscesses; this is the first report of using a hybrid model of PCD and EUS-guided drainage to improve clinical outcome.

We propose a modification of the technique of transmural drainage by adding active aspiration using a wide-bore $10 \mathrm{~F}$ Sohendra dilator and placing a 10F NCD for persistent drainage. This technique is simple and cost effective as compared with placing a FCSEMS. With this technique, we were able to drain the abscess that had ruptured and manage persistent biliary drainage from the abscess cavity.
Our case series included only three patients and all had amoebic liver abscesses. However, we do expect the same results in pyogenic and other causes of liver abscess.

\section{Conclusion}

EUS-guided liver abscess drainage is likely to become the standard of care for abscesses inaccessible to percutaneous drainage. Large multicenter studies comparing EUS-guided transmural drainage using a 10F NCD with a FCSEMS need to be carried out, along with studies comparing outcomes with EUS-guided versus surgical drainage.

\section{Competing interests}

The authors declare that they have no conflict of interest. 


\section{References}

[1] Seewald S, Imazu H, Omar S et al. EUS-guided drainage of hepatic abscess. Gastrointest Endosc 2005; 61: 495-498

[2] Ang T, Seewald S, Teo E et al. EUS-guided drainage of ruptured liver abscess. Endoscopy 2009; 41: E21-E22

[3] Noh S, Park do H, Kim Y et al. EUS-guided drainage of hepatic abscesses not accessible to percutaneous drainage (with videos). Gastrointest Endosc 2010; 71: 1314-1319

[4] Itio T, Ang T, Seewald S et al. Endoscopic ultrasonography-guided drainage for tuberculous liver abscess drainage. Dig Endosc 2011; 23: 158-161

[5] Keohane J, Christopher ], DiMaio C] et al. EUS guided transgastric drainage of caudate lobe liver abscess. J Interv Gastroenterol 2011; 1: 139-141

[6] Koizumi K, Masuda S, Uojima H et al. Endoscopic ultrasound-guided drainage of amoebic liver abscess extending into the hepatic subcapsular space. Clinical J Gastroenterol 2015; 8: 232-235
[7] Tonozuka R, Itoi T, Tsuchiya T et al. EUS-guided drainage of hepatic abscess and infected bilioma using short and long metal stents(with videos). Gastrointest Endosc 2015; 81: 1463-1469

[8] Ogura T, Masuda D, Saori O et al. Clinical outcome of endoscopic ultrasound-guided liver abscess drainage using self-expandable covered metallic stent (with videos). Dig Dis Sci 2016; 61: 303-308

[9] Yamamoto K, Itoi T, Tsuchiya T et al. EUS-guided drainage of hepatic abscess in the right side of the liver of a patient with chilaiditi syndrome. VideoGIE 2017; 2: 299-300

[10] Carbajo AY, BrunieVegas FJ, Garcia Alonso FJ et al. Retrospective cohort study comparing endoscopic ultrasound-guided and percutaneous drainage of upper abdominal abscesses. Dig Endosc 2019; 31 : 431-438

[11] Chin YK, Asok KR. Endoscopic ultrasound-guided drainage of difficultto-access liver abscess. SAGE Open Medicine 2020; 8: 1-6 